\title{
Comparative Effects Between High-intensity Atorvastatin and Rosuvastatin on New Onset Diabetes Mellitus and Cardiovascular Outcome
}

Jah Yeon Choi

Korea University Guro Hospital

Cheol Ung Choi ( $\nabla$ wmagpie@korea.com )

Korea University Guro Hospital

Byoung Geol Choi

Korea University Guro Hospital

Yoonjee Park

Korea University Guro Hospital

Dong Oh Kang

Korea University Guro Hospital

Won Young Jang

Catholic University of Korea St.Vincent Hospital

Woohyeun Kim

Korea University Guro Hospital

Jin Oh Na

Korea University Guro Hospital

Jin Won Kim

Korea University Guro Hospital

Eung Ju Kim

Korea University Guro Hospital

Seung-Woon Rha

Korea University Guro Hospital

Chang Gyu Park

Korea University Guro Hospital

Hong Seog Seo

Korea University Guro Hospital

Myung Ho Jeong

Chonnam National University Hospital

\section{Sung-Chull Chae}

Kyungbook National University Hospital

In-Whan Seong 
Chungnam National University School of Medicine

Chang-Hwan Yoon

Seoul National University Bundang Hospital

Kwang Soo Cha

Pusan National University School of Medicine

\section{Seok Kyu Oh}

Wonkwang University School of Medicine Hospital

\section{Original investigation}

Keywords: acute myocardial infarction, new-onset diabetes mellitus, cardiovascular outcome, atorvastatin, rosuvastatin

Posted Date: June 17th, 2020

DOI: https://doi.org/10.21203/rs.3.rs-35208/v1

License: (c) (i) This work is licensed under a Creative Commons Attribution 4.0 International License. Read Full License 


\section{Abstract}

Background: High-intensity statin is typically used in patients with acute myocardial infarction (AMI) for secondary prevention; however, concern regarding its association with diabetes mellitus has been consistent. We investigated and compared the impact of high-intensity atorvastatin on new-onset diabetes mellitus (NODM) and cardiovascular outcomes with that of high-intensity rosuvastatin for a 3year follow-up period.

Methods: Data from the Korea Acute Myocardial Infarction Registry were collected from November 2011 to October 2015, and 13,104 AMI patients were enrolled from major cardiovascular centers. Among them, 2,221 patients without diabetes who had been administered with high-intensity atorvastatin $(40-80 \mathrm{mg})$ and rosuvastatin $(20 \mathrm{mg})$ were investigated. The atorvastatin and rosuvastatin groups were compared and evaluated for the incidence of NODM and major adverse cardiac event (MACE) including total death, myocardial infarction, and revascularization cases in the following 3 years.

Results: Baseline characteristics were comparable between two groups. Event-free survival rate of NODM and MACE was not significantly different between the atorvastatin and rosuvastatin groups $(92.5 \% \mathrm{vs}$. $90.8 \%$, respectively; Log-rank P-value $=0.550$ ). The event-free survival rate of MACE was also not significantly different between atorvastatin and rosuvastatin groups ( $89.0 \%$ vs. $89.6 \%$, respectively; Log rank p-value $=0.662$ ). Multivariable Cox analysis revealed that the statin type was not a prognostic factor in the development of NODM and MACE.

Conclusions: Administering high-intensity atorvastatin and rosuvastatin in AMI patients produced comparable effects on NODM and clinical outcome, suggesting their clinical equivalence in secondary prevention.

\section{Background}

Statins typically prevent cardiovascular events by lowering total and low-density lipoprotein (LDL) cholesterol levels in the serum. Considering their rapid and sustained clinical advantages, the current guideline recommends administration of high-intensity statin in AMI patients for secondary prevention. However, there has been consistent concern regarding its association with new-onset diabetes mellitus (NODM) [1]. Clinical trials, meta-analyses of randomized controlled trials (RCT), and observational studies have demonstrated a $10-12 \%$ increase in NODM among patients receiving statins [2, 3]. Meta-analysis of 5 large-scale trials comparing intensive and moderate doses of statin have demonstrated that the risk of NODM further increases in intensive therapy group [4]. However, it is unclear if the diabetogenic effect of statins is a class effect. Considering their mandatory role for secondary prevention in acute myocardial infarction (AMI) patients, it would be important to identify the specific statin that can provide protective cardiovascular effects without deteriorating the glycemic status. In Korea, atorvastatin 40-80 mg and rosuvastatin $20 \mathrm{mg}$ are currently available as high-intensity statins for clinical use. Here, we investigated 
and compared the impact of high-intensity atorvastatin and rosuvastatin on cardiovascular outcome and NODM in AMI patients for a 3-year follow-up period.

\section{Methods}

\section{Data collection and study population}

The Korea Acute Myocardial Infarction Registry (KAMIR), a Korean prospective, multicenter, nationwide database supported by the Korean Society of Cardiology, reflects real-world treatment practices and outcomes in Asian patients diagnosed with AMI. Twenty university or community hospitals have participated in the registry. Data collection at the institution level is performed by a well-trained study coordinator using a standardized case report form. The collected data is managed using web-based systems. All participants provided written informed consent, and the study was approved by the ethics committee at each participating institution.

A total of 13,104 AMI patients were enrolled in the KAMIR registry between November 2011 and October 2015. A total of 6,728 patients without a history of diabetes mellitus (DM) with successful percutaneous coronary intervention (PCl) with drug-eluting stent implantation and high-intensity statin treatment were eligible for our study. Patients were selected considering the following exclusion criteria: history of DM or initial $\mathrm{HbA} 1 \mathrm{c}$ level $\geq 6.5 \%$, bare metal stent implantation, plain old balloon angioplasty, or failed PCl. Additionally, we did not include patients with in-hospital major adverse cardiac events (MACE) to specifically include AMI survivors. Finally, 2,221 patients with AMI and high-intensity atorvastatin or rosuvastatin, treatment, according to 2014 ACC/AHA Release Updated Guideline, were enrolled in this study. Of them, $60.7 \%(1,349 / 2,221)$ of patients had received $40-80 \mathrm{mg}$ atorvastatin and $39.3 \%$ $(872 / 2,221)$ had received $20 \mathrm{mg}$ rosuvastatin (Fig. 1).

\section{Clinical outcome and definition}

The primary endpoint was the incidence of NODM and the incidence of MACE during the 3 years of clinical follow-up. Secondary endpoints were each component of MACE, reasons for mortality, MI, and revascularization. Diagnosis of NODM was defined as a $\mathrm{HbA} 1 \mathrm{c}$ level $\geq 6.5 \%$ or newly administration of oral hypoglycemic agents. Clinical data of the enrolled patients were obtained through face-to-face interviews during regular outpatient visits, medical chart reviews, and telephonic interviews.

\section{Statistical analysis}

For continuous variables, differences between the two groups were evaluated by Student's t-test. Data were expressed as mean \pm standard deviation (SD). For discrete variables, differences were expressed as counts and percentages and analyzed using the Chi-square test or Fisher's exact test. The cumulative incidence of NODM and MACE was estimated using the Kaplan-Meier method, and the intergroup differences were determined using the log-rank test. Cox-proportional hazard models were used to 
identify potential prognostic factors for NODM and MACE, and assess the hazard ratio (HR) of the atorvastatin group compared with the rosuvastatin group. Multivariable analysis was performed by including variables with significant $P$-values in the univariate analysis $(P<0.05)$. For all analyses, a $P$ value of $<0.05$ was considered statistically significant. The data were processed using SPSS (version 22.0, Inc Chicago, IL).

\section{Results}

\section{Baseline characteristics}

Baseline clinical, laboratory, and angiographic characteristics are shown in Table 1. We did not observe any significant intergroup differences with regard to age, gender, LV systolic function, incidence of STEMI, and underlying disease such as hypertension, cerebrovascular accidents. Patients in the rosuvastatin group had higher levels of LDL cholesterol and peak CK-MB and a longer total stent length than those in the atorvastatin group. Dual antiplatelet therapy rate was above $99 \%$ in both groups. 
Table 1

Baseline clinical characteristics and angiographic and procedural characteristics

\begin{tabular}{|c|c|c|c|}
\hline Variables & $\begin{array}{l}\text { Atorvastatin } \\
(n=1349)\end{array}$ & $\begin{array}{l}\text { Rosuvastatin } \\
(\mathrm{n}=\mathbf{8 7 2})\end{array}$ & P-value \\
\hline Men & $1105(81.9 \%)$ & $720(82.6 \%)$ & 0.693 \\
\hline Age (years) & $61.0 \pm 12.5$ & $61.0 \pm 12.6$ & 0.918 \\
\hline LV ejection fraction (\%) & $54.1 \pm 9.9$ & $54.0 \pm 9.6$ & 0.906 \\
\hline Body mass index $\left(\mathrm{kg} / \mathrm{m}^{2}\right)$ & $24.5 \pm 3.2$ & $24.4 \pm 3.4$ & 0.442 \\
\hline \multicolumn{4}{|l|}{ Myocardial infarction } \\
\hline ST-segment elevation & $719(53.3 \%)$ & $478(54.8 \%)$ & 0.483 \\
\hline Non-ST-segment elevation & $630(46.7 \%)$ & $394(45.2 \%)$ & \\
\hline Hypertension & $549(40.7 \%)$ & $329(37.7 \%)$ & 0.162 \\
\hline Cerebrovascular accidents & $42(3.1 \%)$ & $29(3.3 \%)$ & 0.781 \\
\hline Total cholesterol (mg/dl) & $195.5 \pm 42.0$ & $196.6 \pm 45.6$ & 0.563 \\
\hline Triglyceride (mg/dl) & $146.5 \pm 120.3$ & $145.4 \pm 139.1$ & 0.852 \\
\hline HDL-cholesterol (mg/dl) & $44.1 \pm 11.1$ & $44.0 \pm 11.1$ & 0.790 \\
\hline LDL-cholesterol (mg/dl) & $126.5 \pm 36.3$ & $130.6 \pm 40.3$ & 0.020 \\
\hline CK-MB (mg/dl) & $125.2 \pm 159.2$ & $139.9 \pm 145.1$ & 0.029 \\
\hline Glucose (mg/dl) & $136.7 \pm 37.4$ & $138.8 \pm 37.9$ & 0.216 \\
\hline Creatinine $(\mathrm{mg} / \mathrm{dl})$ & $0.97 \pm 0.94$ & $0.94 \pm 0.42$ & 0.340 \\
\hline \multicolumn{4}{|l|}{ Discharge medications } \\
\hline Aspirin & $1343(99.6 \%)$ & $865(99.2 \%)$ & 0.280 \\
\hline Clopidogrel & $855(63.4 \%)$ & $473(54.2 \%)$ & $<0.001$ \\
\hline Prasugrel & $188(13.9 \%)$ & $68(7.8 \%)$ & $<0.001$ \\
\hline Ticagreler & $302(22.4 \%)$ & $325(37.3 \%)$ & $<0.001$ \\
\hline Cilostazol & $57(4.2 \%)$ & $29(3.3 \%)$ & 0.283 \\
\hline Calcium channel blockers & $63(4.7 \%)$ & $45(5.2 \%)$ & 0.600 \\
\hline ßblockers & $1181(87.5 \%)$ & $714(81.9 \%)$ & $<0.001$ \\
\hline
\end{tabular}

ACEi, angiotensin-converting enzyme inhibitor; $A R B$, angiotensin receptor blocker; $C K-M B$, Creatine Kinase-MB; HDL, high-density lipoprotein; LDL, low-density lipoprotein; LV, left ventricle 


\begin{tabular}{|llll|}
\hline Variables & $\begin{array}{l}\text { Atorvastatin } \\
(\mathbf{n = 1 3 4 9 )}\end{array}$ & $\begin{array}{l}\text { Rosuvastatin } \\
(\mathbf{n = 8 7 2})\end{array}$ & P-value \\
\hline ACEi & $687(50.9 \%)$ & $321(36.8 \%)$ & $<0.001$ \\
\hline ARB & $426(31.6 \%)$ & $336(38.5 \%)$ & 0.001 \\
\hline Procedural characteristics & $27.6 \pm 12.2$ & $39.8 \pm 13.4$ & $<0.001$ \\
\hline Total stent length (mm) & $1.17 \pm 0.41$ & $1.20 \pm 0.45$ & 0.077 \\
\hline Total stent number & $3.20 \pm 0.44$ & $3.14 \pm 0.44$ & 0.062 \\
\hline Stent diameter (mm) & & & \\
\hline $\begin{array}{l}\text { ACEi, angiotensin-converting enzyme inhibitor; ARB, angiotensin receptor blocker; CK-MB, Creatine } \\
\text { Kinase-MB; HDL, high-density lipoprotein; LDL, low-density lipoprotein; LV, left ventricle }\end{array}$ & \\
\hline
\end{tabular}

\section{Clinical outcomes}

Kaplan-Meier curves for the cumulative incidence of NODM up to 3 years are presented in Fig. 2A and Table 2. There was no significant difference in the event-free survival rate of NODM between the atorvastatin and rosuvastatin groups $(92.5 \%$ vs. $90.8 \%$, respectively; Log-rank P-value $=0.550)$. KaplanMeier curves for the cumulative incidence of MACE up to a period of 3 years are presented in Fig. 2B and Table 2. There was no significant difference between atorvastatin and rosuvastatin groups regarding the event free survival rate of MACE $(89.0 \%$ vs. $89.6 \%$, respectively; Log rank p-value $=0.662)$, reasons for mortality, myocardial infarction, and revascularizations. Comparing $40 \mathrm{mg}$ and $80 \mathrm{mg}$ of atorvastatin with $20 \mathrm{mg}$ of rosuvastatin revealed no significant differences in the event-free survival rate of NODM and MACE (see Additional file 1: Fig. S1A and B). 
Table 2

Cardiovascular Outcomes at 3 Years

\begin{tabular}{|c|c|c|c|}
\hline Clinical outcome & $\begin{array}{l}\text { Atorvastatin } \\
(n=1349)\end{array}$ & $\begin{array}{l}\text { Rosuvastatin } \\
(n=872)\end{array}$ & $p$ value \\
\hline New onset diabetes mellitus & $99(7.5)$ & $70(9.2)$ & 0.550 \\
\hline MACE & $149(11.0)$ & $91(10.4)$ & 0.662 \\
\hline All cause of mortality & $49(3.6)$ & $25(2.9)$ & 0.335 \\
\hline Cardiac death & $19(1.4)$ & $14(1.6)$ & 0.710 \\
\hline Non-cardiac death & $30(2.2)$ & $11(1.3)$ & 0.103 \\
\hline Any myocardial infarction & $30(2.3)$ & $15(1.8)$ & 0.409 \\
\hline STEMI & $8(0.6)$ & $1(0.1)$ & 0.083 \\
\hline NSTEMI & $22(1.7)$ & $14(1.6)$ & 0.956 \\
\hline Any revascularization & $98(7.4)$ & $62(7.2)$ & 0.892 \\
\hline TLR & $24(1.9)$ & $21(2.5)$ & 0.312 \\
\hline TVR & $48(3.7)$ & $34(4.0)$ & 0.692 \\
\hline NTVR & $51(3.9)$ & $31(3.7)$ & 0.788 \\
\hline Stroke & $27(2.0)$ & $10(1.2)$ & 0.127 \\
\hline $\begin{array}{l}\text { MACE, major adverse cardi } \\
\text { NSTEMI, Non-ST-segment } \\
\text { TLR, target lesion revascula }\end{array}$ & $\begin{array}{l}\text { TEMI, ST-se } \\
\text { ial infarctior } \\
\text { get vessel re }\end{array}$ & $\begin{array}{l}\text { evation myoc } \\
\text { non-target ves } \\
\text { ization }\end{array}$ & $\begin{array}{l}\text { retion; } \\
\text { ularizatio }\end{array}$ \\
\hline
\end{tabular}

Potential prognostic factors for NODM were identified via univariate Cox regression analysis. Higher random glucose and triglyceride levels were both significant prognostic factors for NODM in univariable and multivariable analysis. However, the type of statin used was not ( $H R=1.098,95 \%$ confidence interval [CI]: 0.808-1.491, $P=0.551$, Table 3). Conventional risk factor including older age, lower left ventricular ejection fraction (LVEF), and higher creatinine levels were associated with a higher incidence of MACE. The type of high-intensity statin, atorvastatin or rosuvastatin, was not a potential prognostic factor for MACE (HR $=0.944 \%$ confidence interval [Cl]: 0.727-1.225, P = 0.662, Table 3). 
Table 3

Cox regression of clinical outcome

Univariable

Hazard ratio $(95 \% \mathrm{Cl})$
Multivariable

Hazard ratio (95\% Cl)

NODM

\begin{tabular}{llllll} 
Age & $0.988(0.976-1.000)$ & 0.052 & & \\
Male gender & $1.307(0.848-2.013)$ & 0.225 & & \\
\hline Glucose & $1.009(1.006-1.011)$ & $<0.001$ & $1.009(1.006-1.011)$ & $<0.001$ \\
\hline Triglyceride & $1.001(1.000-1.002)$ & 0.011 & $1.001(1.000-1.002)$ & 0.021 \\
\hline B blocker & $0.768(0.517-1.139)$ & 0.189 & &
\end{tabular}

Statin type

$1.098(0.808-1.491)$

0.551

MACE

\begin{tabular}{lllll} 
Age & $1.026(1.016-1.037)$ & $<0.001$ & $1.022(1.0109-1.034)$ & 0.002 \\
\hline Male gender & $0.964(0.695-1.336)$ & 0.824 & & \\
\hline LVEF & $0.974(0.961-0.986)$ & $<0.001$ & $0.979(0.966-0.993)$ & 0.002 \\
\hline Glucose & $1.003(1.000-1.006)$ & 0.089 & & \\
\hline Creatinine & $1.155(1.080-1.235)$ & $<0.001$ & $1.216(1.074-1.377)$ & 0.002 \\
\hline LDL cholesterol & $0.995(0.992-0.999)$ & 0.011 & $0.998(0.994-1.002)$ & 0.265 \\
\hline Statin type & $0.944(0.727-1.225)$ & 0.662 & & \\
\hline ACEi or ARB & $0.761(0.569-1.018)$ & 0.066 & & \\
\hline$\beta$ blocker & $0.855(0.609-1.201)$ & 0.367 & & \\
\hline
\end{tabular}

ACEi, angiotensin-converting enzyme inhibitor; ARB, angiotensin receptor blocker; LDL, low-density lipoprotein; LVEF, left ventricle ejection fraction

\section{Discussion}

To the best of our knowledge, this is the first study to compare the impact of high-intensity statin treatment on the development of NODM and MACE in AMI patients. Our results indicated that highintensity atorvastatin and rosuvastatin therapies showed no significant difference with regard to the incidence of NODM and cardiovascular events.

Statins reduce serum LDL cholesterol level and the risk of cardiovascular events. As numerous studies revealed that the degree of cardiovascular risk reduction is proportional to the statin intensity $[5,6]$, the 
current guideline strongly recommended high-intensity or maximally tolerated intensity statin therapy in AMI patients in the absence of contraindications $[7,8]$. Several studies have that suggested that statin may increase the incidence of $\operatorname{NODM}[3,9]$. The issue has started attracting attention since the Justification for the Use of Statin in Prevention: An Intervention Trial Evaluating Rosuvastatin (JUPITER) trial reported a higher incidence of NODM in patients treated with rosuvastatin for primary prevention [10]. Numerous observational studies [11, 12] and meta-analyses of major RCT [3, 13] have consistently reported an increased incidence of NODM in patients receiving statin treatment. In Korea, a populationbased cohort study using the Korean National Health Insurance claims database has shown an increased incidence of NODM in statin-treated groups [2].

Whether the diabetogenic effect of statin is a class effect has been a controversial subject. Typically, atorvastatin and rosuvastatin are thought to unfavorably influence glycemic parameters, while pitavastatin and pravastatin have relatively neutral effects on glycemic control regardless of the presence or absence of DM [1]. Our group recently published a report regarding the favorable glycemic effects of moderate intensity pitavastatin in comparison to those of moderate intensity atorvastatin and rosuvastatin in AMI patients [14]. However, the current guideline recommends high-intensity or maximally tolerated statin for secondary prevention to achieve cardiovascular improvements in AMI patients [7, 8], and to the best of our knowledge, there is no study that has compared the diabetogenic and cardiovascular effects of different high-intensity statins.

Several studies were conducted regarding the cardiovascular outcome after high-intensity statin therapy. In patients with acute coronary syndrome, both the atorvastatin and rosuvastatin groups had comparable effects on lipid parameters $[15,16]$, although patients with familial hypercholesterolemia in the rosuvastatin group demonstrated a greater reduction in LDL cholesterol levels than those in the atorvastatin group [17]. Some studies has reported more favorable effects of rosuvastatin on reducing atherosclerotic plaque volume $[15,18]$ and plaque stabilization $[15]$ than atorvastatin, however, there has been no significant difference with regard to the cardiovascular outcome in both groups $[17,19]$. In line with previous studies, we could not identify the differences between the effects of high-intensity atorvastatin and rosuvastatin administration on major cardiovascular events.

Several studies have suggested the possible mechanisms underlying the effect of statin on glucose metabolism. Some studies have suggested the interconnection between glucose and lipid metabolisms by demonstrating gene variants affecting glucose metabolism [20-22], cholesterol-dependent conformational change in glucose transporter protein [23], or deleterious effect on islet $\beta$ cells [24] by statins. There is scarce data regarding the mechanisms of different diabetogenic effects of various statins and further study would be needed.

This study has several limitations. First, our study is not an RCT, which inevitably leads to selection bias and an imbalance of baseline characteristics. However, as KAMIR is a prospective registry that enrolls AMI patients in Korean, it can represent real-word clinical data. Second, there is no data on rosuvastatin $40 \mathrm{mg}$ and a relatively small number of patients have been treated with atorvastatin $80 \mathrm{mg}$; hence, dose- 
dependent increases in NODM could not be demonstrated in our study. Third, this study lacks data regarding the compliance of statin during the follow-up period. Despite its limitations, to the best of our knowledge, this is the first study from a multicenter registry that demonstrated detailed real-world data on the effect of high-intensity statin type on incidence of NODM and MACE in AMI patients. Although further studies with prospective randomized trials with a larger sample size are needed, our results provide adequate supportive evidence to compare the diabetogenic and cardioprotective effects of the currently available high-intensity statins.

\section{Conclusions}

In conclusion, high-intensity atorvastatin therapy showed similar incidence of NODM and cardiovascular events when compared with high-intensity rosuvastatin therapy in AMI patients. Although prospective, randomized trials with larger study population are needed to clarify our results, the outcomes presented here provide supportive evidence for the diabetogenic and cardio protective effect of high-intensity statins in AMI patients.

\section{Abbreviations}

AMI: acute myocardial infarction; KAMIR: Korea Acute Myocardial Infarction Registry; LDL: low-density lipoprotein; MACE: major adverse cardiac events; NODM: new-onset diabetes mellitus; PCl: percutaneous coronary intervention; RCT: randomized controlled trials

\section{Declarations}

\section{Ethics approval and consent to participate}

This study was approved by the Institutional Review Board of Korea University (IRB No. 2016GR0740) and all participants provided written informed consent.

\section{Consent for publication}

Not applicable

\section{Availability of data and materials}

We cannot upload the data that has been used in the research because it is confidential

\section{Competing interests}

The authors declare that they have no competing interests. 


\section{Funding}

This research was supported by a fund (2016-ER6304-02) by Research of Korea Centers for Disease Control and Prevention.

\section{Authors' contributions}

Jah Yeon Choi : conception and design of the work, acquisition/analysis/interpretation of data, writingOriginal draft preparation, Byoung Geol Choi : acquisition/analysis/interpretation of data, Yoonjee Park, Dong Oh Kang, Won Young Jang, and Woohyeun Kim : acquisition/analysis of data, Jin Oh Na, Jin Won Kim, Eung Ju Kim, Seung-Woon Rha, Chang Gyu Park and Hong Seog Seo : acquisition of data, Myung Ho Jeong, Sung-Chull Chae, In-Whan Seong, Chang-Hwan Yoon, Kwang Soo Cha, Seok Kyu Oh : conception of the work, acquisition of data, Cheol Ung Choi : conception and design of the work, supervision, reviewing and editing, acquisition/analysis of data.

\section{Acknowledgements}

This study was done with the support of Korean Circulation Society (KCS). The KAMIR study group of the KSC was as follows: Woong Chol Kang, Gachon University Gil Medical Center, Incheon; Kiyuk Chang, Catholic University Hospital, Seoul; Seung Ho Hur, Keimyung University Dongsan Medical Center, Daegu; Seung Woon Rha, Korea University Guro Hospital, Seoul; Kwang-Soo Cha, Pusan National University Hospital, Busan; Hyeon Cheol Gwon, Samsung Medical Center, Seoul; Seok Kyu Oh, Wonkwang University Hospital, Iksan; Jei Keon Chae, Chonbuk National University Hospital, Jeonju; Kyung-Kook Hwang, Chungbuk National University Hospital, Chungju; Chong Jin Kim, East West Neo Medical Center, Seoul; Jung Han Yoon, Wonju Severance Christian Hospital, Wonju; Jin Yong Hwang, Gyeongsang National University Hospital, Jinju; Doo II Kim, Inje University Busan Paik Hospital, Busan; Seung Jae Joo, Jeju National University Hospital, Jeju.

\section{References}

1. Betteridge DJ, Carmena R: The diabetogenic action of statins - mechanisms and clinical implications. Nat Rev Endocrino/2016, 12(2):99-110.

2. Lee J, Noh Y, Shin S, Lim HS, Park RW, Bae SK, Oh E, Kim GJ, Kim JH, Lee S: Impact of statins on risk of new onset diabetes mellitus: a population-based cohort study using the Korean National Health Insurance claims database. Ther Clin Risk Manag 2016, 12:1533-1543.

3. Sattar N, Preiss D, Murray HM, Welsh P, Buckley BM, de Craen AJM, Seshasai SRK, McMurray JJ, Freeman DJ, Jukema JW et al: Statins and risk of incident diabetes: a collaborative meta-analysis of randomised statin trials. The Lancet 2010, 375(9716):735-742. 
4. Preiss D, Seshasai SR, Welsh P, Murphy SA, Ho JE, Waters DD, DeMicco DA, Barter P, Cannon CP, Sabatine MS et al: Risk of incident diabetes with intensive-dose compared with moderate-dose statin therapy: a meta-analysis. Jama 2011, 305(24):2556-2564.

5. Hiro T, Kimura T, Morimoto T, Miyauchi K, Nakagawa Y, Yamagishi M, Ozaki Y, Kimura K, Saito S, Yamaguchi $\mathrm{T}$ et al: Effect of intensive statin therapy on regression of coronary atherosclerosis in patients with acute coronary syndrome: a multicenter randomized trial evaluated by volumetric intravascular ultrasound using pitavastatin versus atorvastatin (JAPAN-ACS [Japan assessment of pitavastatin and atorvastatin in acute coronary syndrome] study). J Am Coll Cardio/ 2009, 54(4):293302.

6. Cannon CP, Steinberg BA, Murphy SA, Mega JL, Braunwald E: Meta-analysis of cardiovascular outcomes trials comparing intensive versus moderate statin therapy. J Am Coll Cardiol 2006, 48(3):438-445.

7. Mach F, Baigent C, Catapano AL, Koskinas KC, Casula M, Badimon L, Chapman MJ, De Backer GG, Delgado V, Ference BA et al: 2019 ESC/EAS Guidelines for the management of dyslipidaemias: lipid modification to reduce cardiovascular risk. Eur Heart J 2020, 41(1):111-188.

8. Grundy SM, Stone NJ, Bailey AL, Beam C, Birtcher KK, Blumenthal RS, Braun LT, de Ferranti S, FaiellaTommasino J, Forman DE et al: 2018 AHA/ACC/AACVPR/AAPA/ABC/ACPM/ADA/AGS/APhA/ASPC/NLA/PCNA Guideline on the Management of Blood Cholesterol: A Report of the American College of Cardiology/American Heart Association Task Force on Clinical Practice Guidelines. J Am Coll Cardio/2019, 73(24):e285-e350.

9. Rajpathak SN, Kumbhani DJ, Crandall J, Barzilai N, Alderman M, Ridker PM: Statin therapy and risk of developing type 2 diabetes: a meta-analysis. Diabetes Care 2009, 32(10):1924-1929.

10. Ridker PM, Pradhan A, MacFadyen JG, Libby P, Glynn RJ: Cardiovascular benefits and diabetes risks of statin therapy in primary prevention: an analysis from the JUPITER trial. The Lancet 2012, 380(9841):565-571.

11. Chen CW, Chen TC, Huang KY, Chou P, Chen PF, Lee CC: Differential impact of statin on new-onset diabetes in different age groups: a population-based case-control study in women from an asian country. PLoS One 2013, 8(8):e71817.

12. Carter AA, Gomes T, Camacho X, Juurlink DN, Shah BR, Mamdani MM: Risk of incident diabetes among patients treated with statins: population based study. BMJ 2013, 346:f2610.

13. Maruyama T, Takada M, Nishibori Y, Fujita K, Miki K, Masuda S, Horimatsu T, Hasuike T: Comparison of Preventive Effect on Cardiovascular Events With Different Statins. Circulation Journal 2011, 75(8):1951-1959.

14. Choi JY, Choi CU, Hwang SY, Choi BG, Jang WY, Kim DY, Kim W, Park EJ, Lee S, Na JO et al: Effect of Pitavastatin Compared with Atorvastatin andRosuvastatin on New-Onset Diabetes Mellitus in PatientsWith Acute Myocardial Infarction. Am J Cardiol 2018, 122(6):922-928.

15. Thondapu V, Kurihara O, Yonetsu T, Russo M, Kim HO, Lee H, Soeda T, Minami Y, Jang IK: Comparison of Rosuvastatin Versus Atorvastatin for Coronary Plaque Stabilization. Am J Cardiol 2019, 
123(10):1565-1571.

16. Altunkeser BB, Tuncez A, Ozturk B, Tezcan H, Ates MS, Yilmaz C, Yalcin MU, Aygul N, Demir K: Comparative effects of high-dose atorvastatin versus rosuvastatin on lipid parameters, oxidized lowdensity lipoprotein, and proprotein convertase subtilisin kexin 9 in acute coronary syndrome. Coron Artery Dis 2019, 30(4):285-290.

17. Perez de Isla L, Arroyo-Olivares R, Muniz-Grijalvo O, Diaz-Diaz JL, Zambon D, Fuentes F, Sanchez Munoz-Torrero JF, Mediavilla JD, Gonzalez-Estrada A, Miramontes-Gonzalez JP et al: Long-term effect of 2 intensive statin regimens on treatment and incidence of cardiovascular events in familial hypercholesterolemia: The SAFEHEART study. J Clin Lipidol 2019, 13(6):989-996.

18. Kumar A, Shariff M, Doshi R: Impact of rosuvastatin versus atorvastatin on coronary atherosclerotic plaque volume - a systematic review and meta-analysis with trial sequential analysis of randomized control trials. Eur J Prev Cardiol 2019:2047487319868035.

19. Perez-Calahorra S, Laclaustra M, Marco-Benedi V, Pinto X, Sanchez-Hernandez RM, Plana N, Ortega E, Fuentes F, Civeira F: Comparative efficacy between atorvastatin and rosuvastatin in the prevention of cardiovascular disease recurrence. Lipids Health Dis 2019, 18(1):216.

20. Schmidt AF, Swerdlow DI, Holmes MV, Patel RS, Fairhurst-Hunter Z, Lyall DM, Hartwig FP, Horta BL, Hyppönen E, Power C et al: PCSK9 genetic variants and risk of type 2 diabetes: a mendelian randomisation study. The Lancet Diabetes \& Endocrinology 2017, 5(2):97-105.

21. Lotta LA, Sharp SJ, Burgess S, Perry JRB, Stewart ID, Willems SM, Luan J, Ardanaz E, Arriola L, Balkau B et al: Association Between Low-Density Lipoprotein Cholesterol-Lowering Genetic Variants and Risk of Type 2 Diabetes: A Meta-analysis. JAMA 2016, 316(13):1383-1391.

22. Ference BA, Robinson JG, Brook RD, Catapano AL, Chapman MJ, Neff DR, Voros S, Giugliano RP, Davey Smith G, Fazio $S$ et al: Variation in PCSK9 and HMGCR and Risk of Cardiovascular Disease and Diabetes. The New England journal of medicine 2016, 375(22):2144-2153.

23. Nowis D, Malenda A, Furs K, Oleszczak B, Sadowski R, Chlebowska J, Firczuk M, Bujnicki JM, Staruch $A D$, Zagozdzon $\mathrm{R}$ et al: Statins impair glucose uptake in human cells. BMJ open diabetes research \& care 2014, 2(1):e000017.

24. Sampson UK, Linton MF, Fazio S: Are statins diabetogenic? Curr Opin Cardio/2011, 26(4):342-347.

\section{Figures}




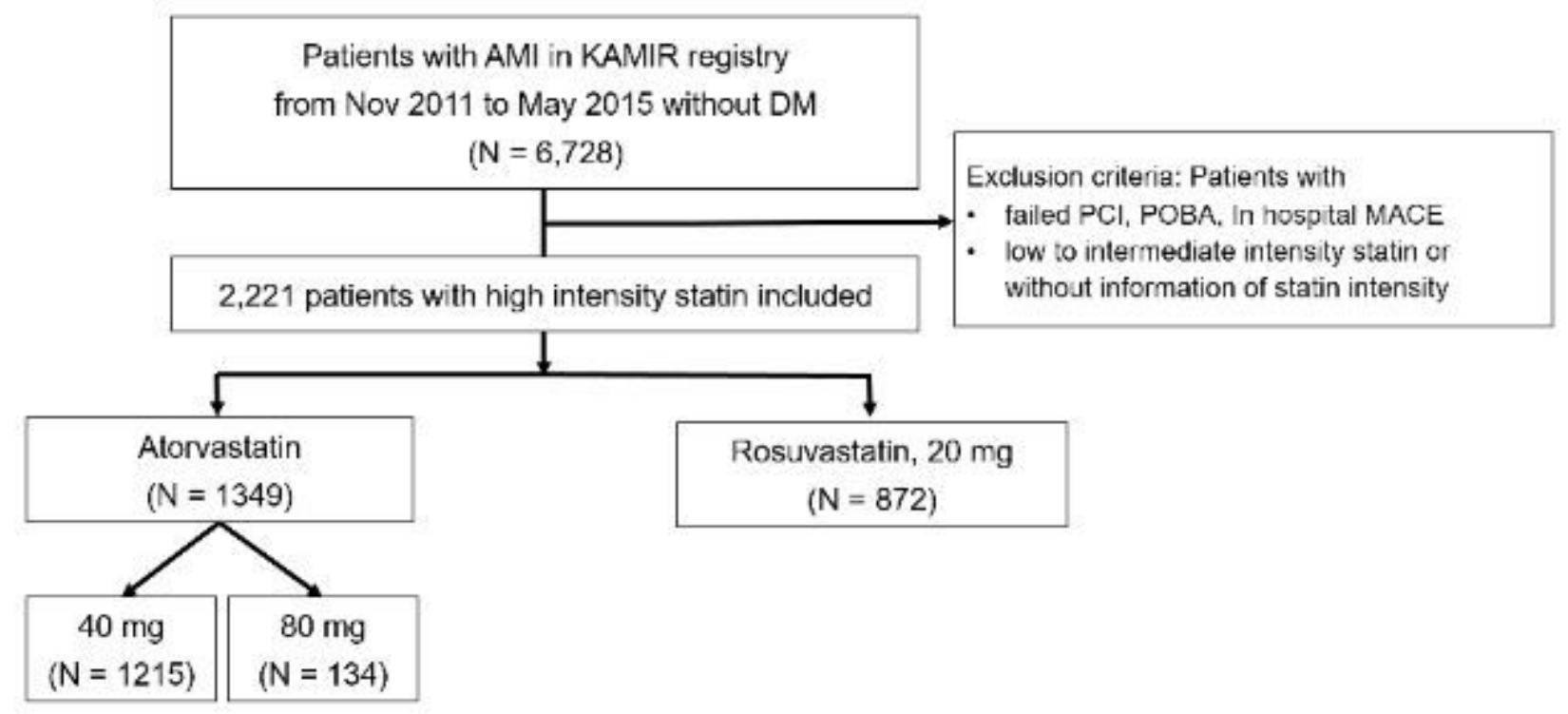

Figure 1

Patient flow chart AMI: Acute myocardial infarction, DM: diabetes mellitus, KAMIR: Korea Acute Myocardial Infarction Registry, MACE: major adverse cardiac events, PCl: percutaneous coronary intervention, POBA: plain old balloon angioplasty

\section{A. New Onset Diabetes Mellitus}

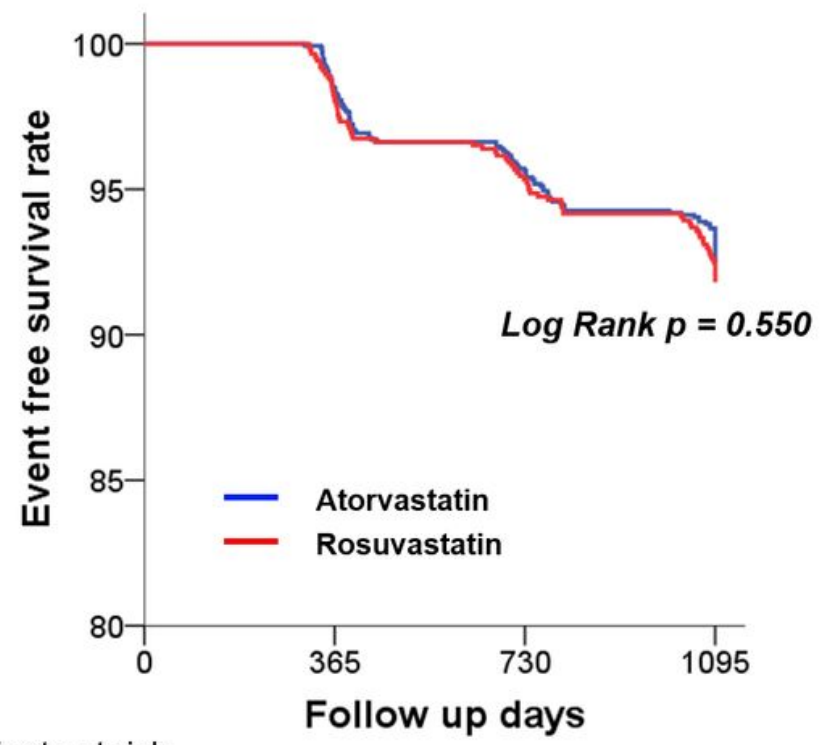

Patients at risk

\section{B. Major Adverse Cardiac Events}

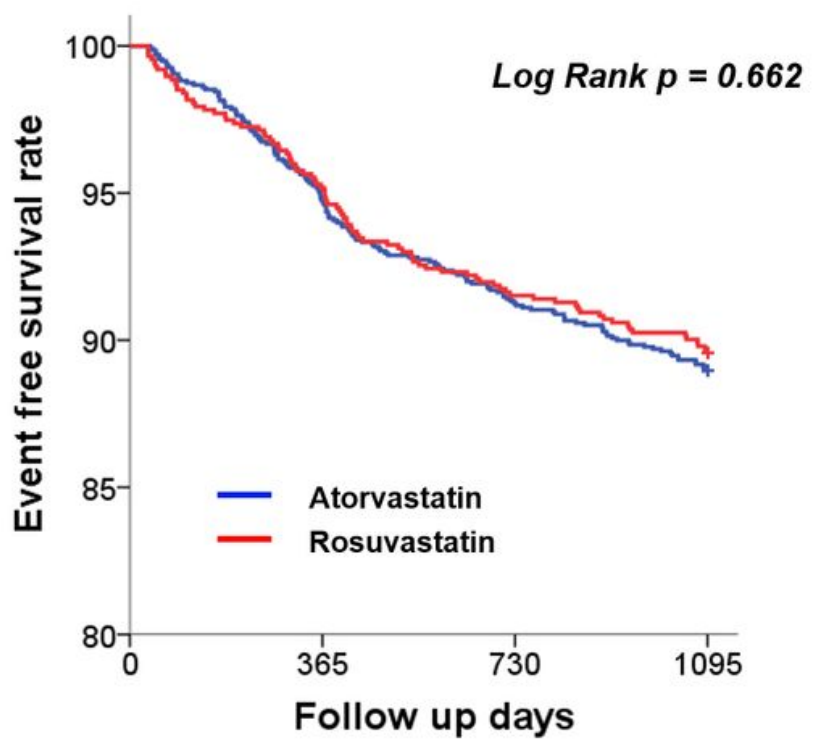

Patients at risk

$\begin{array}{ccccc}1349 & 1328 & 1291 & 1130 \\ - & 873 & 856 & 833 & 803\end{array}$

\section{Figure 2}

Kaplan-Meier curves for event-free survival rate of new-onset diabetes mellitus (A) and major adverse cardiac events (B) according to statin type 


\section{Supplementary Files}

This is a list of supplementary files associated with this preprint. Click to download.

- supplement1.docx 